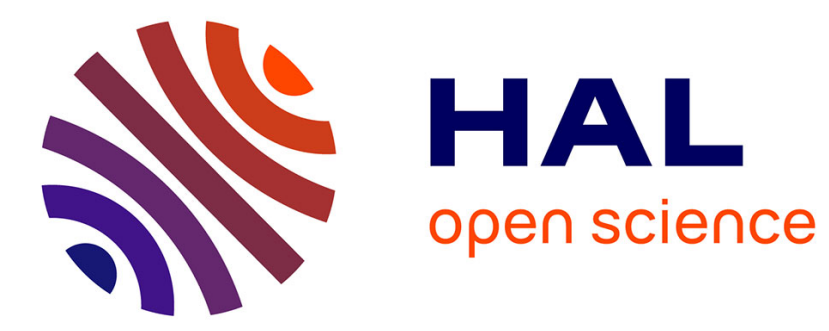

\title{
Étude par une méthode de déphasages des vibrations de surfaces cristallines
}

L. Dobrzynski, G. Leman

\section{To cite this version:}

L. Dobrzynski, G. Leman. Étude par une méthode de déphasages des vibrations de surfaces cristallines. Journal de Physique, 1969, 30 (1), pp.116-124. 10.1051/jphys:01969003001011600 . jpa-00206755

\section{HAL Id: jpa-00206755 https://hal.science/jpa-00206755}

Submitted on 1 Jan 1969

HAL is a multi-disciplinary open access archive for the deposit and dissemination of scientific research documents, whether they are published or not. The documents may come from teaching and research institutions in France or abroad, or from public or private research centers.
L'archive ouverte pluridisciplinaire HAL, est destinée au dépôt et à la diffusion de documents scientifiques de niveau recherche, publiés ou non, émanant des établissements d'enseignement et de recherche français ou étrangers, des laboratoires publics ou privés. 


\title{
ÉTUDE PAR UNE MÉTHODE DE DÉPHASAGES DES VIBRATIONS DE SURFAGES GRISTALLINES
}

\author{
Par LÉONARD DOBRZYNSKI et G. LEMAN, \\ Institut Supérieur d'Électronique du Nord, 3, rue F.-Baes, 59-Lille (France).
}

(Reçu le 22 juillet 1968.)

\begin{abstract}
Résumé. - On se propose de résoudre complètement par une méthode de déphasages le problème des vibrations des surfaces cristallines; on obtient ainsi non seulement des états localisés en surface, s'ils existent, mais aussi des états étendus à tout le cristal, éventuellement résonnants et les fonctions thermodynamiques. La méthode quoique générale est exposée dans le cas d'un cristal cubique simple. Comme application, nous calculons la contribution des phonons aux fonctions thermodynamiques de surface.
\end{abstract}

\begin{abstract}
The crystal surface phonon problem can be completely solved by a phase shift method : one obtains localized surface modes if they exist, and also, eventually, resonant bulk modes and the thermodynamic functions. This general method is developed for a simple cubic crystal. As an application, we calculate the surface phonon thermodynamic functions.
\end{abstract}

L'interaction des phonons avec les surfaces cristallines a déjà fait l'objet de nombreuses études. Plusieurs articles de revue en font la synthèse; citons en particulier ceux de Maradudin [11] et de Ludwig [16].

La méthode des fonctions de Green fut appliquée, pour la première fois, au calcul des vibrations de surface par Lifshitz et al. [1, 2]. Récemment, Brown [3] utilisa cette même approche pour l'étude des états liés et résonnants de phonons et d'électrons, créés par les dislocations et les surfaces.

Nous allons montrer comment, par des considérations de symétrie, la résolution du problème de la surface peut être simplifiée. Nous allons utiliser les déphasages généralisés introduits par de Witt [4] et Toulouse $[4 \mathrm{bis}]$ qui ont ainsi réécrit la formule de trace de Lifshitz [5] sous une forme plus compacte, que nous appliquons dans le cas d'une surface, en suivant une suggestion de Friedel et al. [6].

La méthode est en principe tout à fait générale, mais nous la détaillerons ici dans le cas d'un cristal monoatomique cubique simple, avec interactions centrales entre premiers voisins; les fonctions thermodynamiques de surface ainsi déterminées sont d'un ordre de grandeur tout à fait raisonnable.

I. Résolution formelle. - Soit un cristal monoatomique cubique simple infini et soit $|u\rangle$ le vecteur déplacement des atomes de ce cristal par rapport à leurs positions d'équilibre. Le vecteur $|u\rangle$ a $3 N^{3} \mathrm{com}$ posantes, relatives aux trois directions de l'espace $\sigma=x, y$ et $z$ sur chacun des $N^{3}$ atomes du cristal.
Nous noterons par l'ensemble $(l, m, n)$ d'entiers le site $\mathbf{R}$ de chaque atome :

$$
\mathbf{R}=l \mathbf{a}+m \mathbf{b}+n \mathbf{c}
$$

où $\mathbf{a}, \mathbf{b}$ et $\mathbf{c}$, de module $a$, sont les trois vecteurs de base du réseau.

I.1. CRistal infini. - Dans l'approximation harmonique, l'énergie potentielle s'écrit :

$$
V=\frac{1}{2}\left\langle u\left|D_{0}\right| u\right\rangle
$$

où $D_{0}$ est la matrice dynamique du problème [1]. Remarquons que les éléments de matrice $D_{0}$ du problème ne dépendent que des différences $\mathbf{R}-\mathbf{R}^{\prime}$ entre les différents sites, ainsi que des directions $\sigma$ et $\sigma^{\prime}$.

Étant donné que nous aurons la symétrie de translation dans le plan de la surface, nous pouvons poser, pour une surface (001) :

$$
|u\rangle=\frac{1}{M^{1 / 2}}|v\rangle \mathrm{e}^{i\left(l \varphi_{1}+m \varphi_{2}-\omega t\right)}
$$

où $M$ est la masse des atomes et $\varphi_{1}, \varphi_{2}, \varphi_{3}$ sont les projections du vecteur $\boldsymbol{\varphi}=\mathbf{k} a$ sur les trois axes (k étant le vecteur de propagation) $x, y$ et $z$; $\omega$ et $t$ représentent la pulsation angulaire et le temps; le vecteur $|v\rangle$ à $3 N$ composantes, relatives aux trois directions $\sigma=x, y$ et $z$ et à chacun des plans d'atomes perpendiculaires à la direction $z$.

A l'aide de (3), le problème devient unidimensionnel. Les équations du mouvement s'écrivent en effet :

$$
\left(D-\omega^{2}\right)|v\rangle=0 .
$$


Les éléments de matrice de $D$ s'écrivent :

$$
D_{n}^{\sigma \sigma^{\prime}}=\frac{1}{M} \sum_{l, m} D_{0 R}^{\sigma \sigma^{\prime}} \mathrm{e}^{-i\left(l \varphi_{1}+m \varphi_{2}\right)} .
$$

La résolution bien connue de l'équation (4) donne les valeurs propres :

$$
\omega^{2}=\omega_{j}^{2}\left(\varphi_{3}\right) \quad(j=1,2,3)
$$

et les vecteurs propres orthonormés correspondants :

$$
\left|v_{j}\left(\varphi_{3}\right)\right\rangle=N^{-1 / 2} \sum_{n, \sigma} f_{j}^{\sigma}\left(\varphi_{3}\right) \mathrm{e}^{i n \varphi_{3}}|n, \sigma\rangle
$$

$N^{3}$ étant le nombre d'atomes du cristal et le ket $|n, \sigma\rangle$ rappelle que $|v\rangle$ a des composantes suivant $\sigma$ pour chaque $n$ donné.

I.2. Surface libre. - Pour obtenir deux surfaces libres, nous coupons le réseau infini en deux par le plan :

$$
z=a / 2
$$

et nous supprimons les interactions entre les atomes se trouvant de part et d'autre de ce plan.

Ce modèle néglige le déplacement possible des atomes de surface par rapport aux positions d'équilibre qu'ils auraient dans un cristal infini, ainsi que toute modification en surface des forces de liaison interatomiques.

Par analogie avec (4), nous avons maintenant à résoudre :

$$
\left(D+\Lambda-\omega^{2}\right)|w\rangle=0
$$

où $|w\rangle$ est le vecteur propre correspondant à $|v\rangle$ pour le cristal infini et $\Lambda$ est la différence entre la matrice dynamique du cristal avec ces deux surfaces libres et celle du cristal infini.

Écrivons la fonction propre solution de l'équation (9) sous la forme :

$$
|w\rangle=N^{-1 / 2} \sum_{n, \sigma} U_{n}^{\sigma}|n, \sigma\rangle .
$$

Ce modèle de surface libre ainsi construit est invariant par translation d'ensemble du cristal comme l'ont remarqué Lifshitz et al. [1] dont nous nous sommes inspirés jusqu'ici.

I.3. MÉThode DEs DÉPhasages. - La solution générale de (9) est la somme de la solution de l'équation sans second membre et d'une solution particulière de l'équation avec second membre :

$$
|w\rangle=|v\rangle+\Lambda G_{0}^{+}|w\rangle
$$

où :

$$
|v\rangle=\sum_{j}\left|v_{j}\left(\varphi_{3}\right)\right\rangle
$$

et :

$$
G_{0}^{+}\left(\omega^{2}\right)=\frac{1}{\omega^{2}-D+i \varepsilon}
$$

$\varepsilon$ étant un infiniment petit positif.

a) Si $\omega$ est en dehors des bandes $\omega_{j}\left(\varphi_{3}\right)$ du cristal infini, (11) se réduit à :

$$
|w\rangle=\Lambda G_{0}^{+}|w\rangle .
$$

Quand on remplace dans l'équation (14) $|w\rangle$ par son expression (10), on obtient un système infini d'équation homogène en $U_{n}^{\sigma}$ :

$$
U_{n}^{\sigma}=\sum_{n^{\prime} \sigma^{\prime}}\left(\Lambda G_{0}^{+}\right)_{n n^{\prime}}^{\sigma \sigma^{\prime}} U_{n^{\prime}}^{\sigma^{\prime}}
$$

Pour que ce système admette une solution, il faut donc que :

$$
\text { dét }\left(I-\Lambda G_{0}^{+}\right)=0
$$

où $I$ est la matrice unité.

En reportant (15) dans (10) et en utilisant la condition de normalisation de $|w\rangle$ :

$$
\langle w \mid w\rangle=1
$$

nous pouvons déterminer $|w\rangle$, la fonction propre correspondante à la valeur propre $\omega^{2}$ donnée par (16) et qui représente un état localisé.

Remarquons que tout l'exposé, à partir de l'équation (3), a été fait à $\varphi_{1}$ et $\varphi_{2}$ donnés. Nous avons omis d'écrire explicitement ces variables pour alléger l'écriture. Donc l'équation (16) peut donner un ou plusieurs états liés pour chaque couple $\left(\varphi_{1}, \varphi_{2}\right)$; d'où l'apparition des bandes d'ondes de surface $\omega\left(\varphi_{1}, \varphi_{2}\right)$. Nous avons d'autre part trois bandes $\omega_{j}\left(\varphi_{1}, \varphi_{2}, \varphi_{3}\right)$ d'ondes de volume.

Précisons encore que (16) peut donner un état lié pour $\omega$ en dehors des bandes $\omega_{j}\left(\varphi_{3}\right)$ à $\left(\varphi_{1}, \varphi_{2}\right)$ donnés, à ne pas confondre avec les bandes $\omega_{j}\left(\varphi_{1}, \varphi_{2}, \varphi_{3}\right)$ d'ondes de volume.

b) Si $\omega$ est à l'intérieur des bandes $\omega_{j}\left(\varphi_{3}\right),|w\rangle$ ne diffère de $|v\rangle$ que par un déphasage que nous allons préciser.

La généralisation au cas d'une surface des résultats obtenus pour une impureté ponctuelle [4-5] est immédiate.

Utilisons la notation :

$$
H=\text { dét }\left(1-\Lambda G_{0}^{+}\right)
$$

$H$ est fonction de $\omega^{2}$ et dépend des deux paramètres $\varphi_{1}$ et $\varphi_{2}$.

$H$, déjà analysé en termes des représentations irréductibles du groupe des translations le long de la surface, pourra aussi être analysé en termes des représentations irréductibles $\Gamma$ du groupe ponctuel de $\Lambda$. Il suffira d'étudier $H$ dans chacune de ces représentations $\Gamma$. Nous aurons :

$$
H_{\Gamma}=\left|H_{\Gamma}\right| \mathrm{e}^{-i \eta} \Gamma \text {. }
$$

L'annulation de la partie réelle de $H_{\Gamma}$ :

$$
\mathrm{ReH}_{\Gamma}=0
$$

donnera une résonance de symétrie $\Gamma$ dans la bande des états continus $\omega_{j}^{2}\left(\varphi_{3}\right)$.

La somme de tous les déphasages partiels $\eta_{\Gamma}$ sera :

$$
\eta=\sum_{\Gamma \varphi_{1}, \varphi_{2}} \mathrm{~d}_{\Gamma} \eta_{\Gamma}
$$

où $\mathrm{d}_{\Gamma}$ indique le nombre de fois qu'une représentation irréductible $\Gamma$ apparaît dans $\Lambda$.

c) Soit $F$ une fonction thermodynamique quelconque du gaz de phonons. Dans l'approximation 
harmonique, $F$ peut être écrite comme une somme sur tous les états $q$ du système :

$$
F=\sum_{q} f\left(\omega_{q}^{2}\right) .
$$

On peut considérer $(22)$ comme la définition de $f\left(\omega_{q}^{2}\right)$.

La variation de $F$ due à l'introduction des surfaces libres s'écrira facilement grâce à la généralisation de la formule de trace de Lifshitz [5] donnée dans le cas d'une impureté ponctuelle et en utilisant les déphasages généralisés définis par Toulouse [4 bis]; on obtient :

$$
\Delta F=-\frac{1}{\pi} \int \mathrm{d} \omega^{2} f^{\prime}\left(\omega^{2}\right) \eta\left(\omega^{2}\right)
$$

avec :

$$
f^{\prime}\left(\omega^{2}\right)=\mathrm{d} f / \mathrm{d} \omega^{2} .
$$

En utilisant les déphasages généralisés, (23) comprendra la contribution des états localisés sortis des bandes $\omega_{j}^{2}\left(\varphi_{3}\right)$.

Notons encore [5] que la variation de la densité d'états s'écrit :

$$
\Delta n\left(\omega^{2}\right)=\frac{1}{\pi} \frac{\mathrm{d} \eta}{\mathrm{d} \omega^{2}} .
$$

Ainsi la méthode des déphasages permet de résoudre rigoureusement et complètement le problème de la surface dans le cadre du modèle choisi. Les autres méthodes précédemment utilisées $[11,16]$ pour le calcul des états de surface ne permettaient pas de déterminer directement la variation de la densité d'états des ondes de volume et d'en déduire les fonctions thermodynamiques de surface; celles-ci ont cependant été étudiées directement par d'autres méthodes [11] à basse et haute températures.

Pour illustrer cette méthode des déphasages, nous allons maintenant l'appliquer à un modèle simple.

II. Application à un modèle simple. - Nous allons retenir seulement les interactions centrales entre premiers voisins. Pour ce modèle de cristal, il n'est pas possible de satisfaire simultanément les conditions pour la stabilité élastique $\left(C_{11}>0, C_{44}>0, C_{11}-C_{12}>0\right.$, $C_{11}+2 C_{12}>0$ ) et les conditions sur les constantes de force venant de ce que l'énergie potentielle du cristal doit être invariante pour des rotations infinitésimales de l'ensemble du corps. Cependant, ce modèle est fort intéressant pour avoir une approche simple des phénomènes de surface.

II.1. DÉTERmination Des DÉPhasages. - L'équation du mouvement dans la direction $x$ de l'atome situé au site $\mathbf{R}$ (éq. (1)) s'écrit en notant par $X_{1}$ et $X_{2}$ le rapport des constantes de force par la masse $M$ des atomes :

$$
\begin{aligned}
-\omega^{2} u_{l, m, n}^{x}= & X_{1}\left(u_{l+1, m, n}^{x}+u_{l-1, m, n}^{x}-2 u_{l, m, n}^{x}\right) \\
& +X_{2}\left(u_{l, m+1, n}^{x}+u_{l, m-1, n}^{x}\right. \\
& \left.+u_{l, m, n+1}^{x}+u_{l, m, n-1}^{x}-4 u_{l, m, n}^{x}\right) .
\end{aligned}
$$

Les équations du mouvement dans les directions $y$ et $z$ s'obtiennent à partir de (26) par permutation circulaire de $x, y, z$ et des accroissements de $l, m, n$.

D'où les valeurs propres de la matrice $D$ :

$\omega_{1}^{2}=2 X_{1}\left(1-\cos \varphi_{1}\right)+2 X_{2}\left(2-\cos \varphi_{2}-\cos \varphi_{3}\right)$

$\omega_{2}^{2}=2 X_{1}\left(1-\cos \varphi_{2}\right)+2 X_{2}\left(2-\cos \varphi_{1}-\cos \varphi_{3}\right)$

$\omega_{3}^{2}=2 X_{1}\left(1-\cos \varphi_{3}\right)+2 X_{2}\left(2-\cos \varphi_{1}-\cos \varphi_{2}\right)$

et les vecteurs propres correspondants :

$$
\mathbf{f}_{1}=\left(\begin{array}{l}
1 \\
0 \\
0
\end{array}\right) ; \quad \mathbf{f}_{2}=\left(\begin{array}{l}
0 \\
1 \\
0
\end{array}\right) ; \quad \mathbf{f}_{3}=\left(\begin{array}{l}
0 \\
0 \\
1
\end{array}\right) .
$$

A) Symétries du problème de la surface libre. - Pour un cristal cubique simple, la première zone de Brillouin du cristal infini est un cube de côté $2 \pi$. Étant donné que nous n'avons plus que la symétrie de translation dans les directions de $\varphi_{1}$ et $\varphi_{2}$, il nous faudra considérer l'intersection par le plan $\varphi_{3}=0$ de la zone de Brillouin à trois dimensions. Et « la zone de Brillouin » à deux dimensions que nous aurons à considérer est donnée par la figure 1.

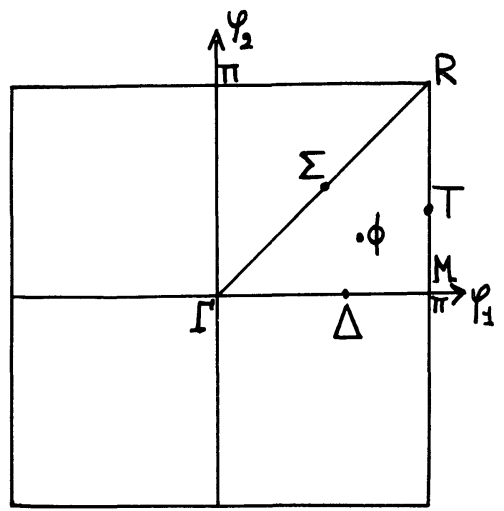

Frg. 1. - "Zone de Brillouin à deux dimensions " d'un cubique simple.

En plus de la symétrie de translation parallèle au plan de la surface, notre problème présente des symétries ponctuelles que nous nous proposons d'étudier maintenant.

La matrice $D$, comme on l'a vu précédemment, est celle d'un problème unidimensionnel. Il en est de même de $\Lambda$ exprimée dans la base des $|n, \sigma\rangle . \Lambda$ comme $D$ est invariante par les opérations du groupe ponctuel (fig. 2) :

$$
D_{4 h}=D_{4} \times i
$$

avec les notations de Tinkham [7]. Ce groupe est équivalent à :

$$
C_{4 v} \times \sigma_{h} .
$$

Afin de simplifier la discussion, nous utiliserons dans ce qui suit ce dernier produit direct plutôt que le premier. 
Par symétrie, il suffit d'étudier le quadrant $\Gamma \mathrm{MR}$ de la zone de Brillouin à deux dimensions (fig. 1).

Pour un point quelconque $\Phi$ de ce quadrant, le groupe ponctuel se réduit à $C_{1 h}$. Les fonctions de base des représentations irréductibles $\Phi_{2}$ et $\Phi_{1}$ respectivement symétrique et antisymétrique sont du type :

$$
\frac{|0 x\rangle+|1 x\rangle}{\sqrt{2}} ; \frac{|0 y\rangle+|1 y\rangle}{\sqrt{2}} ; \frac{|0 z\rangle-|1 z\rangle}{\sqrt{2}}
$$

et :

$$
\frac{|0 x\rangle-|1 x\rangle}{\sqrt{2}} ; \frac{|0 y\rangle-|1 y\rangle}{\sqrt{2}} ; \frac{|0 z\rangle+|1 z\rangle}{\sqrt{2}} \quad \begin{gathered}
\text { pour } \Phi_{2} .
\end{gathered}
$$

Il ne reste plus qu'à étudier $C_{4 v}$ pour les points et les axes de haute symétrie de la «zone de Brillouin à deux dimensions " (fig. 1). Il est facile de voir que, pour les axes $\Delta, \Sigma$ et $T, C_{4 v}$ se réduit à $C_{1 v}$. Pour le point $\mathrm{M}$, nous avons $C_{2 v}$; et pour les points $R$ et $\Gamma, C_{4 v}$.

Une discussion semblable des symétries d'une surface a été faite par Jones [8].

B) Déphasages. - Pour obtenir $H$ (éq. (18)), nous calculons en appendice les matrices $\Lambda$ et $G_{0}^{+}$et finalement $H$ s'écrit sous la forme :

$$
H=\left|\begin{array}{ll}
H_{\mathrm{S}} & \\
& H_{\mathrm{AS}}
\end{array}\right|
$$

les indices $\mathrm{S}$ et AS caractérisant respectivement les éléments symétriques et antisymétriques par rapport au plan $z=a / 2$. Ces expressions de $H_{\mathrm{S}}$ et $H_{\mathrm{AS}}$ sont :

$$
\begin{gathered}
H_{\mathrm{S}}=\left|\begin{array}{lll}
1 & & \\
& 1 & \\
& & H_{\mathrm{S}}^{z}
\end{array}\right| \\
H_{\mathrm{AS}}=\left|\begin{array}{lll}
H_{\mathrm{AS}}^{x} & & \\
& H_{\mathrm{AS}}^{y} & \\
& & 1
\end{array}\right|
\end{gathered}
$$

avec :

$$
\begin{gathered}
H_{\mathrm{S}}^{z}=\left(\delta_{3}^{\mathrm{ex}}-i \delta_{3}^{\mathrm{in}}\right)\left|\frac{\omega^{2}-\alpha_{3}-\beta_{3}}{\omega^{2}-\alpha_{3}+\beta_{3}}\right|^{1 / 2} \\
H_{\mathrm{AS}}^{x}=\left(\delta_{1}^{\mathrm{ex}}-i \delta_{1}^{\mathrm{in}}\right)\left|\frac{\omega^{2}-\alpha_{1}-\beta_{1}}{\omega^{2}-\alpha_{1}+\beta_{1}}\right|^{1 / 2} \\
H_{\mathrm{AS}}^{y}=\left(\delta_{2}^{\mathrm{ex}}-i \delta_{2}^{\mathrm{in}}\right)\left|\frac{\omega^{2}-\alpha_{2}-\beta_{2}}{\omega^{2}-\alpha_{2}+\beta_{2}}\right|^{1 / 2}
\end{gathered}
$$

où nous avons utilisé les notations suivantes :

$\delta_{j}^{\text {in }}=1$ pour $\omega$ à l'intérieur de la bande $\omega_{j}\left(\varphi_{3}\right)$ $\delta_{j}^{\text {in }}=0$ pour $\omega$ à l'extérieur de la bande $\omega_{j}\left(\varphi_{3}\right)$

$$
\begin{gathered}
\delta_{j}^{\mathrm{ex}}=1-\delta_{j}^{\mathrm{in}} \\
\omega_{j}^{2}\left(\varphi_{3}\right)=\alpha_{j}+\beta_{j} \cos \varphi_{3} \quad(j=1,2,3) .
\end{gathered}
$$

L'équation (19) nous donne trois déphasages partiels différents de zéro :

$$
\begin{aligned}
\eta_{\mathrm{S}}^{z} & =\frac{\pi}{2} \delta_{3}^{\text {in }} \\
\eta_{\mathrm{AS}}^{x} & =\frac{\pi}{2} \delta_{1}^{\text {in }} \\
\eta_{\mathrm{AS}}^{y} & =\frac{\pi}{2} \delta_{2}^{\text {in }} .
\end{aligned}
$$

Remarquons que les fonctions propres (7) écrites dans la base (29) des fonctions symétriques (S) et antisymétriques (AS) par rapport au plan $z=a / 2$ ont des composantes proportionnelles à :

$$
f_{j}^{x} \cos \frac{n \varphi_{3}}{2} ; \quad f_{j}^{y} \cos \frac{n \varphi_{3}}{2} ; \quad f_{j}^{z} \sin \frac{n \varphi_{3}}{2}
$$

dans le cas (S) et :

$$
f_{j}^{x} \sin \frac{n \varphi_{3}}{2} ; \quad f_{j}^{y} \sin \frac{n \varphi_{3}}{2} ; \quad f_{j}^{z} \cos \frac{n \varphi_{3}}{2}
$$

dans le cas (AS).

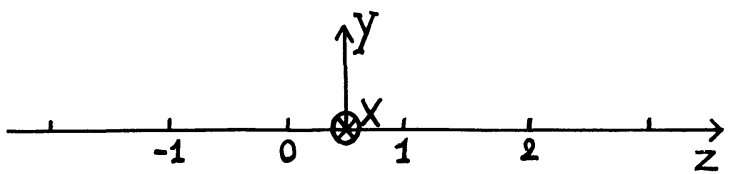

FIG. 2.

Aspect unidimensionnel du problème des surfaces libres.

Les déphasages (39-41), dus à l'introduction des deux surfaces libres, transforment les composantes (42) et (43) en une même forme symétrique de propagation:

$$
f_{j}^{\sigma} \cos \frac{n \varphi_{3}}{2}
$$

par rapport au plan de coupure $z=a / 2$. Ce résultat est logique, car maintenant nos deux demi-cristaux n'ont plus d'interactions entre eux et l'état vibrationnel est le même dans ces deux demi-cristaux.

C) Résonances de surface. - En utilisant la forme des déphasages généralisés (39-41) ainsi que la relation (24), nous voyons que dans ce modèle un demiétat sort du haut des trois bandes $\omega_{j}^{2}\left(\varphi_{3}\right)$ pour venir se placer en bas de ces bandes. Nous obtenons donc trois bandes de résonances de surface :

$$
\omega_{j}^{2}\left(\varphi_{1}, \varphi_{2}\right)=\alpha_{j}+\beta_{j} \quad(j=1,2,3)
$$

soit, en utilisant (27) et (38) :

$$
\begin{aligned}
& \omega_{1}^{2}\left(\varphi_{1}, \varphi_{2}\right)=2 X_{1}\left(1-\cos \varphi_{1}\right)+2 X_{2}\left(1-\cos \varphi_{2}\right) \\
& \omega_{2}^{2}\left(\varphi_{1}, \varphi_{2}\right)=2 X_{2}\left(1-\cos \varphi_{1}\right)+2 X_{1}\left(1-\cos \varphi_{2}\right) \\
& \omega_{3}^{2}\left(\varphi_{1}, \varphi_{2}\right)=2 X_{2}\left(2-\cos \varphi_{1}-\cos \varphi_{2}\right) .
\end{aligned}
$$

Si l'on tient compte de constantes de force autres que centrales, on peut s'attendre à ce que ces résonances (46) se détachent du bas des bandes $\omega_{j}\left(\varphi_{3}\right)$ pour devenir des états localisés de surface. 
II.2. Fonctions thermodynamiques DE SURfaGe. - Le déphasage total s'écrit ici :

$$
\eta=\frac{\pi}{2} \sum_{\varphi_{1}, \varphi_{2}} \sum_{j} \delta_{j}^{\text {in }} .
$$

A partir de là, en utilisant les équations (22-24), nous pouvons calculer la contribution des vibrations cristallines aux fonctions thermodynamiques de surface. Pour l'énergie libre $F$, la quantité $f$ définie par (22) s'écrit en fonction de la température absolue $T$ et des constantes $\hbar$ de Planck et $k$ de Boltzmann :

$$
f=\frac{\hbar \omega}{2}+k T \log \left[1-\exp \left(-\frac{\hbar \omega}{k T}\right)\right] .
$$

L'entropie $S$, l'énergie $E$ et la chaleur spécifique $C_{\mathrm{v}}$ à volume $V$ constant peuvent en être déduites à partir des relations thermodynamiques ci-dessous :

$$
\begin{aligned}
S & =-\left(\frac{\partial F}{\partial T}\right)_{V} \\
E & =F+T S \\
C_{\mathrm{v}} & =\left(\frac{\partial E}{\partial T}\right)_{V} .
\end{aligned}
$$

Grâce à la forme particulièrement simple (39-41) des déphasages, nous pouvons obtenir les fonctions thermodynamiques, en portant (47) dans (23) et en interchangeant l'ordre des sommations, sous la forme :

$\Delta F=-\frac{1}{2} \frac{N^{2}}{(2 \pi)^{2}} \sum_{j} \int_{-\pi}^{+\pi} \mathrm{d} \varphi_{1} \int_{-\pi}^{+\pi} \mathrm{d} \varphi_{2} \int_{0}^{\infty} \mathrm{d} \omega^{2} f^{\prime}\left(\omega^{2}\right) \delta_{j}^{\text {in }}$

soit :

$$
\begin{aligned}
\Delta F=\frac{N^{2}}{2 \pi^{2}} \sum_{j} \int_{0}^{+\pi} \mathrm{d} \varphi_{1} \\
\quad \int_{0}^{+\pi} \mathrm{d} \varphi_{2} f\left(\alpha_{j}-\left|\beta_{j}\right|\right)-f\left(\alpha_{j}+\left|\beta_{j}\right|\right) .
\end{aligned}
$$

A) Résultats à basses températures $T$ par rapport à la température de Debye $T_{\mathrm{D}}$. $-\mathrm{A}$ basses températures, seules les faibles valeurs de l'énergie sont excitées et nous obtenons à partir de (53), après un peu d'algèbre, la contribution par unité de surface des phonons aux fonctions thermodynamiques de la surface (001) :

$$
\begin{aligned}
\Delta C_{\mathrm{v}} & =K T^{2} \\
\Delta S & =\frac{K T^{2}}{2} \\
\Delta F & =-\frac{K T^{3}}{6}
\end{aligned}
$$

avec :

$$
K=\frac{3}{4 \pi} \frac{1}{C_{\mathrm{t}}^{2}}(1+2 \sqrt{m}) \zeta(3) \frac{k^{3}}{\hbar^{2}}
$$

où $\zeta(3)=1,202$ est la constante dzéta de Riemann; les vitesses transverses $C_{\mathrm{t}}$ et longitudinales $C_{1}$ du son sont ici telles que :

$$
\begin{aligned}
& C_{\mathrm{t}}^{2}=X_{2} a^{2} \\
& C_{1}^{2}=X_{1} a^{2}
\end{aligned}
$$

et :

$$
m=X_{2} / X_{1}=C_{44} / C_{11} .
$$

Les $C_{\mathrm{ij}}$ sont les constantes élastiques avec les notations habituelles.

Maradudin et al. [9], après un calcul assez long, obtiennent pour une surface (001) d'un cristal cubique simple isotrope à la limite des grandes longueurs d'onde :

$$
K=\frac{5}{2 \pi} \frac{1}{C_{\mathrm{t}}^{2}} \zeta(3) \frac{k^{3}}{\hbar^{2}} .
$$

La dépendance en $T$ de ces résultats est valable pour toute surface car elle provient de la symétrie de translation suivant deux directions. La valeur de $K$ variera bien sûr si nous considérons d'autres surfaces que (001).

\begin{tabular}{|c|c|c|}
\hline \multicolumn{3}{|c|}{ VALEURS DE $\Delta C_{\mathrm{v}} \exp \cdot / \Delta C_{\mathrm{v}}$ (GALGuléE) } \\
\hline & $\begin{array}{c}\text { DupUIS } \\
\text { ET MARADUDIN } \\
(\mathrm{AVEC} \\
\left.C_{1}^{2}=3 C_{\mathrm{t}}^{2}\right) \\
-\end{array}$ & $\begin{array}{c}\text { NOTRE } \\
\text { TRAVAII } \\
-\end{array}$ \\
\hline & 3,8 & 3,21 \\
\hline & 1,5 & 0,98 \\
\hline $\mathrm{NaCl}$ (échant & 2 & 2,02 \\
\hline
\end{tabular}

La comparaison avec les résultats expérimentaux a été faite par Dupuis et al. [10] et par Maradudin [11]. Le tableau I donne le résultat de Maradudin et al. [9] et le nôtre comparé aux valeurs expérimentales [12-13]. Nous avons retenu pour les constantes $C_{44}$ et $C_{11}$ à $T=0$ les valeurs utilisées aussi par Dupuis et al. $[10]$ :

pour $\mathrm{MgO}$ :

$$
\begin{aligned}
& C_{44}=15,68 \times 10^{11} \text { dynes } \times \mathrm{cm}^{-2} \\
& C_{11}=29,9 \times 10^{11} \text { dynes } \times \mathrm{cm}^{-2}
\end{aligned}
$$

pour $\mathrm{NaCl}$ :

$$
\begin{aligned}
& C_{44}=1,327 \times 10^{11} \text { dynes } \times \mathrm{cm}^{-2} \\
& C_{11}=5,750 \times 10^{11} \text { dynes } \times \mathrm{cm}^{-2} .
\end{aligned}
$$

\section{TABLEAU I}

L'accord de nos valeurs calculées avec les valeurs expérimentales est relativement bon étant donné la simplicité du modèle.

B) Chaleur spécifique pour $T / T_{\mathrm{D}} \gg 1$. - Toujours grâce à la forme particulièrement simple (39-41) des déphasages, nous pouvons aussi calculer algébriquement la chaleur spécifique de surface pour $T / T_{\mathrm{D}} \gg 1$.

En portant dans (53) la quantité $f$ correspondant 
à $C_{\mathrm{v}}$ déduite de (48) et en faisant un développement limité pour $\hbar \omega / k T \ll 1$, nous avons obtenu :

$\frac{\Delta G_{\mathrm{v}}(T)}{3 N^{3} k}=-\sum_{n=1}^{\infty}(-)^{n} B_{2 n} \frac{(1-2 n)}{2 n !}\left(\frac{\hbar}{k T}\right)^{2 n} K_{2 n}$

où $B_{2 n}$ représentent les nombres de Bernoulli et :

$K_{2 n}=-\frac{1}{24 \pi^{2}} \frac{1}{N} \sum_{j} \int_{-\pi}^{+\pi} \mathrm{d} \varphi_{1}$

$$
\int_{-\pi}^{+\pi} \mathrm{d} \varphi_{2}\left[\left(\alpha_{j}+\left|\beta_{j}\right|\right)^{n}-\left(\alpha_{j}-\left|\beta_{j}\right|\right)^{n}\right]
$$

ce qui donne par unité de surface, dans le cas où $X_{1}=X_{2}=X$ :

$\Delta C_{\mathrm{v}}(T)=\frac{3}{2} \frac{k}{a^{2}}\left[\frac{1}{72}\left(\frac{T_{\mathrm{D}}}{T}\right)^{2}-\frac{1}{1440}\left(\frac{T_{\mathrm{D}}}{T}\right)^{4}+\ldots\right]$

en utilisant :

$$
\omega_{\mathrm{M}}^{2}=12 X
$$

et :

$$
k T_{\mathrm{D}}=\hbar \omega_{\mathrm{M}} .
$$

Comme $\Delta C_{\mathrm{v}}$ tend vers zéro quand $T$ augmente, cette quantité sera tout à fait négligeable devant la chaleur spécifique de volume à haute température $\left(C_{\mathrm{v}}=3 N^{3} k\right)$.

Le calcul de l'entropie de surface pour $T / T_{\mathrm{D}} \gg 1$ peut être fait aussi de cette manière, mais en recourant cependant à une intégration numérique.

Ici aussi la dépendance en $T$ de ces résultats est la même quelle que soit la surface considérée, autre que (001).

G) Résultats en fonction de $T$ pour $X_{1}=X_{2}$ dans un cas particulier. - Les fonctions thermodynamiques à hautes températures par rapport à $T_{\mathrm{D}}$ peuvent être obtenues [14] aisément grâce à un développement en série dont le premier terme est celui que donne l'approximation d'Einstein. Par la méthode des déphasages, nous pouvons obtenir le résultat exact, par calcul numérique. Nous avons fait ce calcul à toutes températures, quoique nous ne connaissons pas de données expérimentales sur la variation avec $T$ des fonctions thermodynamiques. Nous avons d'abord calculé $\eta$ (47), puis $\Delta F(23)$.

Prenons des valeurs numériques correspondantes à $\mathrm{MgO}$ :

$$
\begin{gathered}
C_{11}=29,9 \times 10^{11} \text { dynes } \times \mathrm{cm}^{-2} \\
C_{12}=8,56 \times 10^{11} \text { dynes } \times \mathrm{cm}^{-2} \\
C_{44}=15,68 \times 10^{11} \text { dynes } \times \mathrm{cm}^{-2} \\
M=3,34 \times 10^{-23} \mathrm{~g} \\
a=2,102 \times 10^{-8} \mathrm{~cm}
\end{gathered}
$$

$M$ étant la valeur moyenne des masses de $\mathrm{Mg}$ et de O.
Nous en tirons avec :

$$
\begin{aligned}
& 2 X=X_{1}+X_{2}=\frac{a}{M}\left(C_{11}+C_{44}-3 C_{12}\right) \\
& 2 X=0,1249 \times 10^{28} \text { c.g.s. }
\end{aligned}
$$

La figure 3 donne le déphasage total $\eta$ (éq. (47)); la variation de densité d'état $\Delta n$ par atome de surface

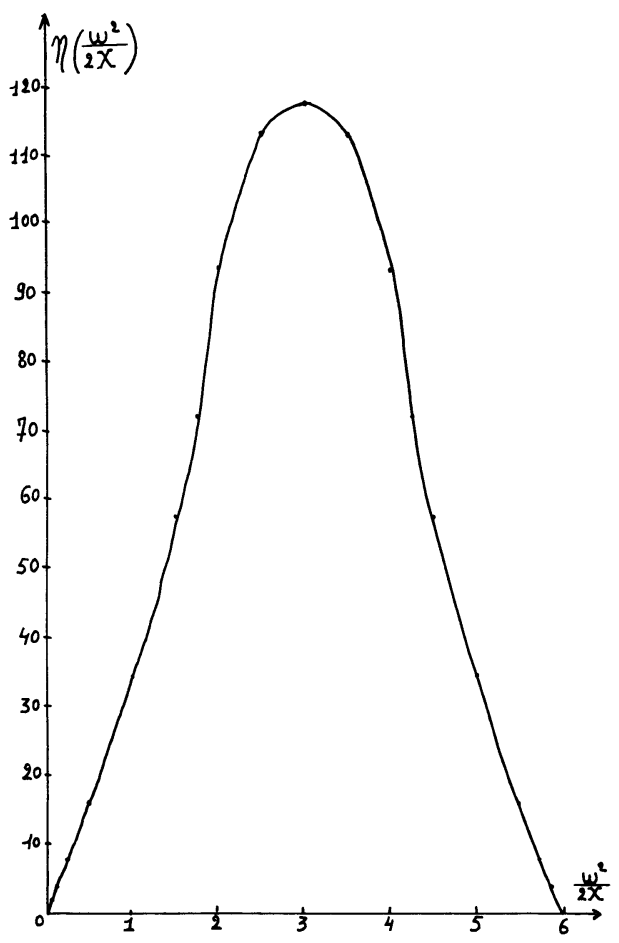

FIG. 3. - Déphasage total $\eta$, par atome de surface, en fonction de $\omega^{2} / 2 X$.

Erratum : En ordonnées, lire $: \eta\left(\frac{\omega^{2}}{2 X}\right) / 2 \pi^{2}$.

et en fonction de $\omega^{2} / 2 X$ s'obtient à partir de (25).

Les figures 4 et 5 représentent respectivement les courbes $\Delta S(T)$ et $\Delta C_{v}(T)$ de l'entropie et de la chaleur spécifique, par unité de surface.

a) Nous retrouvons le fait que $\Delta C_{v}$ tend vers zéro quand la température augmente; ce résultat est en accord avec ce que donne un développement en moments de densité d'état. Pour un cristal infini [17] :

$\frac{C_{v}(T)}{3 N^{3} k}=1-\sum_{n=1}^{\infty}(-)^{n} B_{2 n} \frac{(1-2 n)}{2 n !}\left(\frac{\hbar}{k T}\right)^{2 n} \mu_{2 n}$

avec :

$$
\mu_{2 n}=\frac{1}{3 N^{3}} \operatorname{Tr}\left(D_{0}\right)^{n} \text {. }
$$

La variation $\Delta \mu_{2 n}$ des moments de densité d'état peut être évaluée, par une méthode de décompte de chemins à travers le cristal, utilisée par Cyrot-Lackman [15] pour le calcul de l'énergie de surface des métaux de transition. Cette méthode donne aisément les $\Delta \mu_{2 n}$ pour petits $n$. Nous avons ainsi retrouvé 


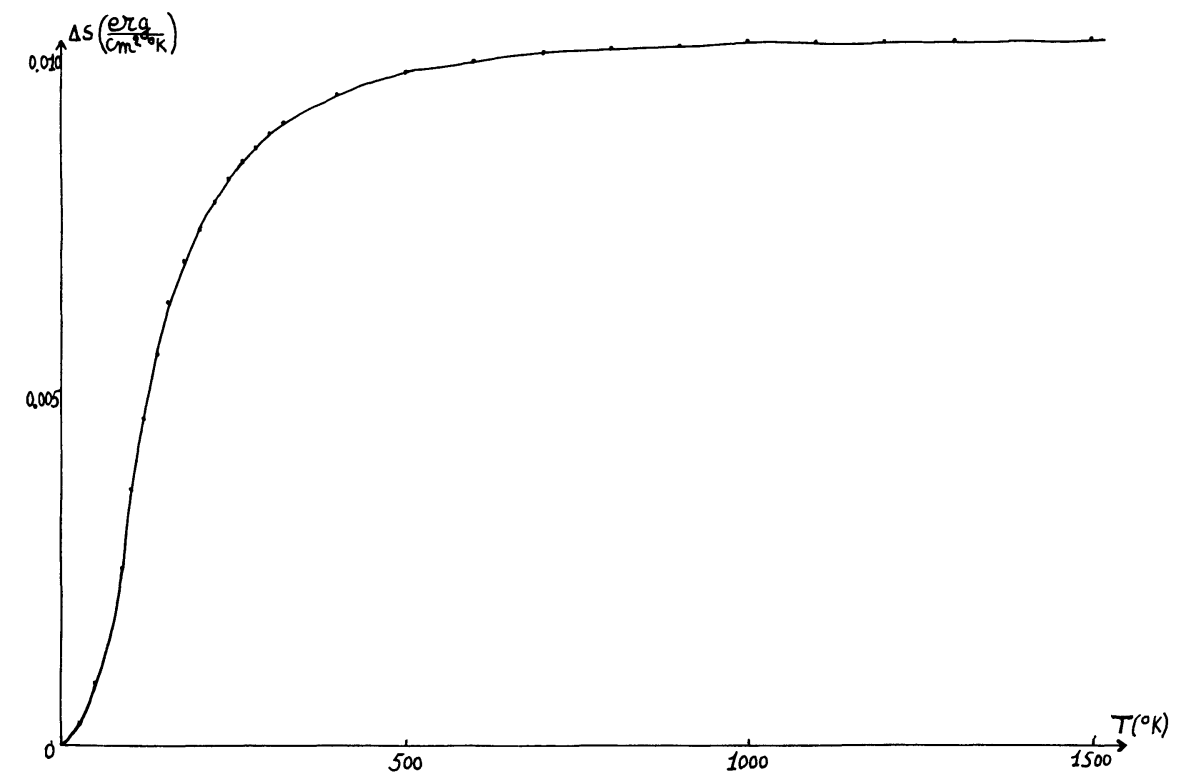

FIG. 4. - Courbe de $\Delta S(T)$ par unité de surface.

Erratum : L,es nombres inscrits en ordonnées doivent être multipliés par 10.

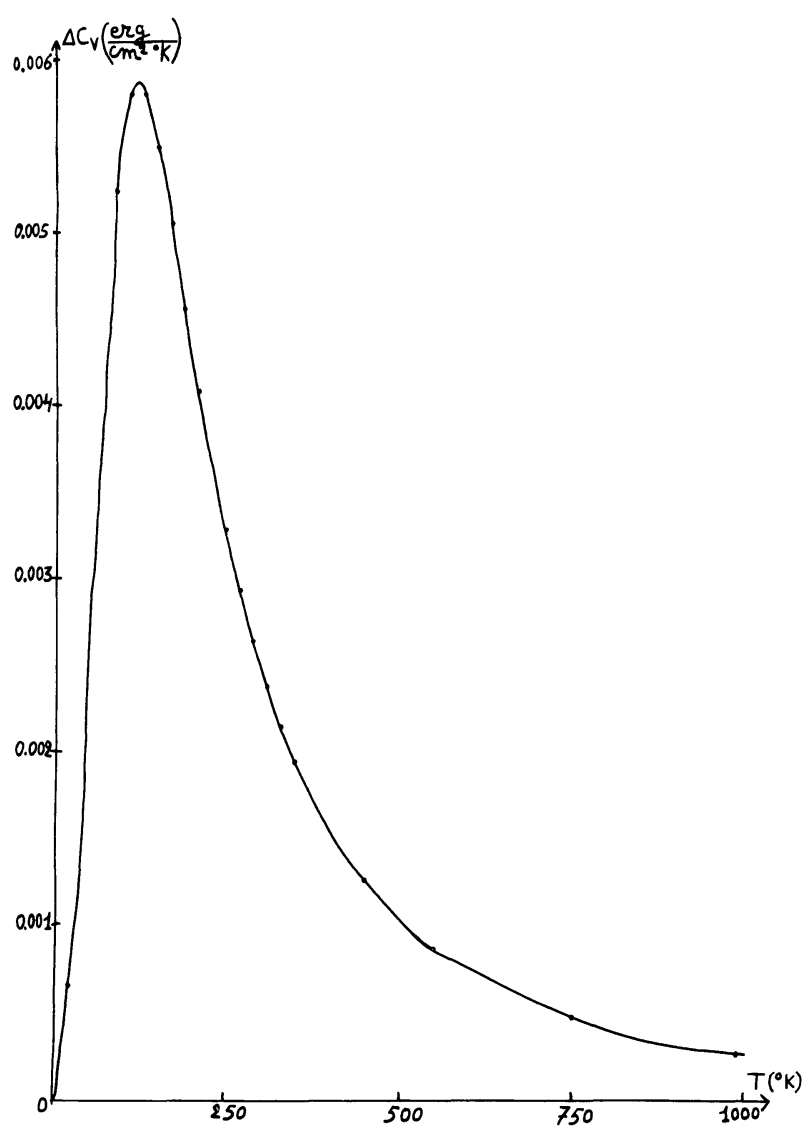

Fig. 5. - Courbe de $\Delta C_{v}(T)$ par unité de surface. Erratum : Les nombres inscrits en ordonnées doivent être multipliés par 10. exactement le même résultat (65) que par la méthode des déphasages.

Remarquons que $\Delta C_{v}$ passe par un maximum ( $\simeq 0,059 \mathrm{erg} /{ }^{\circ} \mathrm{K} \cdot \mathrm{cm}^{2}$ ) pour $T \simeq 120^{\circ} \mathrm{K}$, alors que nous avons ici $T_{D} \simeq 660 \mathrm{o}$. La valeur de $T$ pour laquelle apparaît le maximum peut, a priori, être différente pour des surfaces autres que (001). En écrivant que pour la température correspondant à ce maximum la chaleur spécifique de volume par atome est :

$$
C_{v}=3 k / A
$$

où $A$ est une constante sans dimension pouvant valoir quelques unités et en appelant $q$ le rapport du nombre d'atomes total au nombre d'atomes en surfaces, nous avons ici pour une seule surface (001) :

$$
\Delta C_{v} / C_{v}=0,063 A / q \text {. }
$$

La valeur numérique $\operatorname{de} A$ et $q$ dépend du type d'échantillons que l'on peut imaginer : si l'on imagine qu'on puisse mesurer la chaleur spécifique d'une seule couche d'atomes déposée sur un substrat, on aurait $q=1$ et en supposant $A=4$, on aurait par exemple dans ce cas très particulier :

$$
\Delta C_{v} / C_{v} \simeq 0,25
$$

b) Comparons le résultat obtenu à haute température pour l'entropie de surface, par une « méthode de moments »[14], soit :

$\Delta S=(0,852+0,075+\ldots) 10^{-1} \mathrm{erg} /{ }^{\circ} \mathrm{K} . \mathrm{cm}^{2}$.

Nous constatons ainsi que l'approximation d'Einstein est bonne à $17 \%$ près dans ce cas, et si on tient compte du premier terme correctif on a une précision de $10 \%$. 
Conclusion. - Nous avons montré que le problème d'une surface libre peut être résolu complètement par la méthode des déphasages de la même façon que le problème d'une impureté ponctuelle.

Des considérations de symétrie peuvent simplifier la résolution. En utilisant un modèle très simple pour décrire les vibrations des atomes, nous avons obtenu les modes étendus et les fonctions thermodynamiques de surface à hautes températures. Un seul de ces résultats semble avoir été obtenu jusqu'ici par d'autres auteurs : la chaleur spécifique à basses températures $[9,11]$. Nous avons pu en particulier estimer avec quelle précision l'approximation d'Einstein permet de calculer [14] l'entropie de surface à haute température.

Dans ce modèle de cristal cubique simple avec interactions centrales entre premiers voisins, étant donné la forme particulièrement simple des déphasages (39-41), nous pouvons aussi résoudre exactement le problème de défauts en surface. Ceci est en principe toujours possible, mais conduirait avec des modèles plus élaborés à des calculs numériques assez longs, sauf peut-être dans certains cas limites où le développement en série des fonctions de Green est possible.

De plus, la méthode des déphasages est en principe aussi riche en applications pour les problèmes de surfaces et de dislocations que pour les problèmes d'impuretés ponctuelles; elle s'applique formellement de la même façon pour l'étude des vibrations cristallines, des ondes de spin et des électrons en liaisons fortes.

Remerciements. - Nous remercions vivement le Professeur J. Friedel qui nous a donné l'idée de ce travail et l'a suivi tout au long de son exécution.

L'un d'entre nous (L. D.) tient à remercier le $\mathrm{D}^{\mathrm{r}} \mathrm{G}$. Toulouse pour de nombreuses et fructueuses discussions.

\section{APPENDICE}

Calcul de $H=\operatorname{dét}\left(I-\Lambda G_{0}^{+}\right)$. - A.1. Matrice. $\Lambda$. - Les équations du mouvement (26) écrites sous la forme (4) sont :

$$
\begin{aligned}
-\omega^{2} v_{n}^{x}=X_{1} & \left(2 \cos \varphi_{1}-2\right) v_{n}^{x} \\
& +X_{2}\left(2 v_{n}^{x} \cos \varphi_{2}+v_{n+1}^{x}+v_{n-1}^{x}-4 v_{n}^{x}\right)
\end{aligned}
$$

L'équation pour $v_{n}^{y}$ s'obtient à partir de l'équation en $v_{n}^{x}$ en changeant $x$ en $y$ et $\varphi_{1}$ en $\varphi_{2}$.

Nous en tirons $\Lambda$ (éq. (9)), qui est ici diagonale quels que soient $\varphi_{1}$ et $\varphi_{2}$ dans la base des fonctions (29) :

$$
\begin{gathered}
\Lambda=\left|\begin{array}{ll}
\Lambda_{\mathrm{s}} & \\
& \Lambda_{\mathrm{As}}
\end{array}\right| \\
\Lambda_{\mathrm{s}}=\left|\begin{array}{lll}
0 & \\
& 0 & \\
& -2 X_{1}
\end{array}\right| \\
\Lambda_{\mathrm{As}}=\left|\begin{array}{lll}
-2 X_{2} & -2 X_{2} & \\
& & 0
\end{array}\right| .
\end{gathered}
$$

A.2. Matrice des fonctions de Green $G_{0}^{+}$. Les éléments de matrice de $G_{0}^{+}$dans la base des $|n, \sigma\rangle$ s'écrivent de façon tout à fait générale :

$G_{0}^{+\sigma \sigma_{n^{\prime}}^{\prime}}\left(\omega^{2}\right)=\frac{1}{2 \pi} \sum_{j} \int \mathrm{d} \varphi_{3} \frac{f_{j}^{\sigma} f_{j}^{\sigma^{\prime}} \mathrm{e}^{i\left(n-n^{\prime}\right) \varphi_{3}}}{\omega^{2}-\omega_{j}^{2}\left(\varphi_{3}\right)+i \varepsilon}$

pour $\varphi_{3}$ variant à l'intérieur de la première zone de Brillouin du cristal infini.

Pour évaluer les parties réelles et imaginaires des éléments de matrice de $G_{0}^{+}$, nous utilisons la formule bien connue :

$$
\frac{1}{x+i \varepsilon}=\operatorname{PP}\left(\frac{1}{x}\right)-i \pi \delta(x)
$$

où $\operatorname{PP}\left(\frac{1}{x}\right)$ désigne la partie principale de $\frac{1}{x}$ et $\delta(x)$ la « fonction » delta.

Nous pouvons écrire (A.5) dans la base des fonctions (29) sous la forme :

$$
G_{0}^{+}\left(\omega^{2}\right)=\frac{1}{\pi} \sum_{j} \int_{0}^{\pi} \mathrm{d} \varphi_{3} \frac{A_{j}\left(\varphi_{3}\right)}{\omega^{2}-\omega_{j}^{2}\left(\varphi_{3}\right)+i \varepsilon} .
$$

Pour écrire la matrice $A_{j}\left(\varphi_{3}\right)$, nous avons tenu compte du fait que les termes, sous le signe somme de (A.7), antisymétriques en $\varphi_{3}$, donnent un résultat nul par intégration de $-\pi$ à $+\pi$ :

$$
A_{j}=\left|\begin{array}{ll}
A_{j \mathrm{~s}} & \\
& A_{j \mathrm{As}}
\end{array}\right|
$$

avec :

$$
\begin{aligned}
A_{j \mathrm{~s}} & =\mid \begin{array}{ll}
\left(f_{j}^{x}\right)^{2}\left(1+\cos \varphi_{3}\right) & f_{j}^{x} f_{j}^{y}\left(1+\cos \varphi_{3}\right) \\
f_{j}^{x} f_{j}^{y}\left(1+\cos \varphi_{3}\right) & \left(f_{j}^{y}\right)^{2}\left(1+\cos \varphi_{3}\right)
\end{array} \\
A_{j \mathrm{As}} & =\mid \begin{array}{ll}
\left(f_{j}^{x}\right)^{2}\left(1-\cos \varphi_{3}\right) & f_{j}^{x} f_{j}^{y}\left(1-\cos \varphi_{3}\right) \\
f_{j}^{x} f_{j}^{y}\left(1-\cos \varphi_{3}\right) & \left(f_{j}^{y}\right)^{2}\left(1-\cos \varphi_{3}\right)
\end{array}
\end{aligned}
$$


Tous calculs faits, nous avons obtenu :

$$
G_{0}^{+}=\left|\begin{array}{ll}
G_{0 \mathrm{~s}}^{+} & \\
& G_{0 \mathrm{As}}^{+}
\end{array}\right|
$$

avec :

$$
G_{0 \mathrm{~s}}^{+}=\left|\begin{array}{ccc}
0 & & \\
& 0 & \\
& & G_{0 \mathrm{~s}}^{+z}
\end{array}\right|
$$

et :

$$
G_{0 \mathrm{As}}^{+}=\left|\begin{array}{lll}
G_{0 \mathrm{As}}^{+x} & & \\
& G_{0 \mathrm{As}}^{+y} & \\
& & 0
\end{array}\right|
$$

et :

$G_{0 \Gamma}^{+}=\frac{1}{\beta_{j}}\left[1-\left(\delta_{j}^{\mathrm{ex}}+i \operatorname{Sgn} \beta_{j} \delta_{j}^{\mathrm{in}}\right)\left|\frac{\omega^{2}-\alpha_{j}-\beta_{j}}{\omega^{2}-\alpha_{j}+\beta_{j}}\right|^{1 / 2}\right]$

avec $j$ prenant respectivement les valeurs 3,1 et 2 lorsque $\Gamma$ représente ${ }_{\mathrm{s}}^{z},{ }_{\mathrm{As}}^{x}$ et ${ }_{\mathrm{As}}^{y}$.

$\operatorname{Sgn} \beta_{j}$ est écrit pour le signe de $\beta_{j}$ et les $\delta_{j}^{\text {in }}$ et $\delta_{j}^{\text {ex }}$ sont définis par (36) et (37).

A.3. Matrice $H$. - A partir de (A.2-A.4) et de (A.11-A.14), nous obtenons $H$ donné par (30-35).

\section{BIBLIOGRAPHIE}

[1] LIFshitz (I. M.) et Rosenzweig (L. N.), Izvest. Akad. Nauk. SSSR, Ser. Fiz., 1948, 12, 667.

[2] Rosenzweig (L. N.), Uchenye Zapiski Hark. Gosudarst. Univ. Trudy Fiz. Mat. Otdel., 1950, 2, 19.

[3] Brown (R. A.), Phys. Rev., 1967, 156, 889.

[4] DE WITT (B. S.), Phys. Rev., 1956, 103, 1565, section III.

[4 bis] Toulouse (G.), Solid State Comm., 1966, 4, 593.

[5] Lifshitz (I. M.), Nuovo Cim., 1956, Suppl. 4, 7716.

[6] Friedel (J.), Gautier (F.), Gomes (A. A.) et LENGLART (P.), Quant. Theor. Atoms, 1966, p. 445.

[7] Tinkhay (M.), Group Theory and Quantum Mechanics, McGraw-Hill Book Company.

[8] Jones (R. O.), Proc. Phys. Soc., 1966, 89, 443.

[9] Maradudin (A. A.) et Wallis (R. F.), Phys. Rev., 1966, 148, 945 .
[10] Dupuis (M.), Mazo (R.) et ONSAGER (L.), J. Chem. Phys., 1960, 33, 1452.

[11] Maradudin (A. A.), Solid State Physics, 1966, 19, 100 .

[12] Giauque (W. F.) et Archibald (R. C.), J. Am. Chem. Soc., 1937, 59, 561.

[13] PatTerson (D.), Morrisson (J. A.) et Thompson (F. W.), Can. J. Chem., 1955, 33, 240.

[14] Dobrzynski (L.) et Frieder. (J.), Surface Science, 1968, 5, 65.

[15] Cyro't-Lackman (F.), J. Phys. Chem. Solids, 1968, 29, 1235.

[16] Ludwig (W.), Springer Tracts in Modern Physics, Ergebnisse der exakten Naturwissenschaften (Éditeur : G. Hohler), 1967, 43, 206.

[17] Maradudin (A. A.), Solid State Physics, 1963, Suppl. 3, 213. 